\title{
Time-resolved and parameter-free measures of spike train synchrony: properties and applications
}

\author{
Mario Mulansky ${ }^{*}$, Nebojsa Bozanic, Thomas Kreuz \\ From 24th Annual Computational Neuroscience Meeting: CNS*2015 \\ Prague, Czech Republic. 18-23 July 2015
}

The relevance of exact spike timings in neural coding was presumed since a long time and has now been experimentally established, see e.g. in [1,2]. A popular approach to the analysis of spike timings is to measure the synchrony of spike trains. With the recent advancements of the experimental techniques, it is now possible to simultaneously record the activity of hundreds of neurons. The analysis of such collective responses requires new mathematical tools that are able to detect synchrony in groups of spike trains. Here, we present three methods to quantify spike train synchrony that are applicable in such multivariate situations. All of these methods are parameter-free and time-resolved which makes them easy to handle and able to detect temporal changes of synchrony.

Specifically, we discuss the ISI-distance [3], the SPIKEdistance [4] and the very recently proposed SPIKE-Synchronization [5]. The ISI-distance is based on the relative differences of interspike intervals, while the SPIKEdistance uses exact spike timings. SPIKE-Synchronization can be understood as a time-resolved, spike-wise coincidence detector. Figure 1 shows exemplarily the timeresolved profiles of all three methods for 50 artificially created spike trains.

We analyze the mathematical properties of all three measures and discuss their advantages and disadvantages [6]. Specific focus lies on the statistical relevance of the obtained values compared to random spike trains. By calculating the expectation values for Poisson spike trains we are able to provide an important point of reference for interpreting numerical and experimental results. Finally, we show exemplary applications of the methods to spike trains obtained from numerical simulations [7] as well as experimental recordings [4].

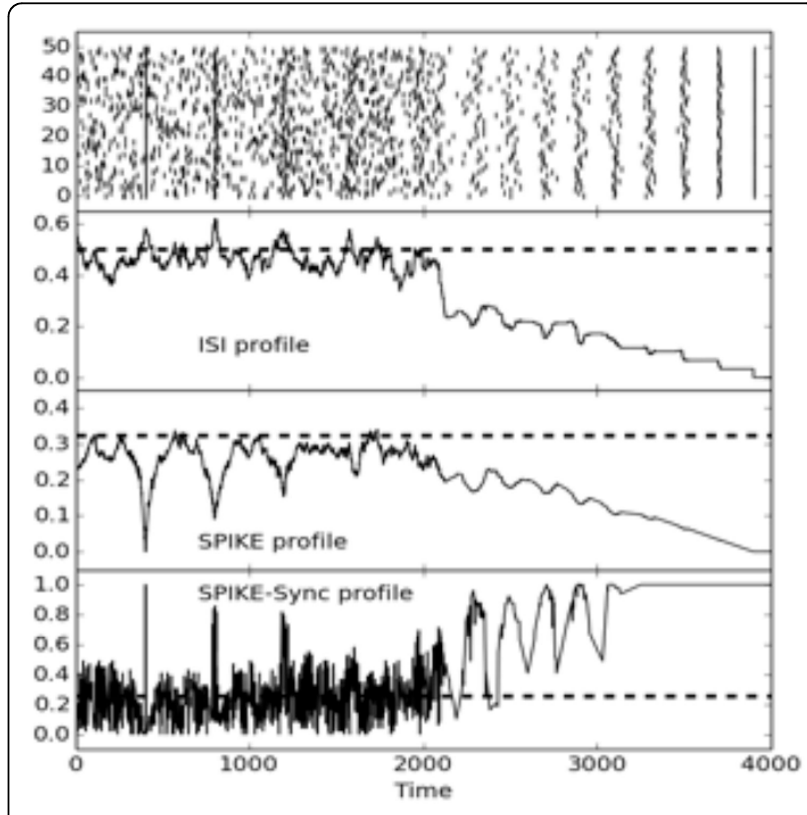

Figure 1 Multivariate ISI, SPIKE and SPIKE-Synchronization profiles for $\mathbf{5 0}$ artificially generated spike trains. The dashed lines represent the respective expectation values for random Poisson spike trains.

The methods are implemented in both the Matlabbased graphical user interface SPIKY [5] and the Python library PySpike.

\section{Acknowledgements \\ This work was supported by the European Commission through the Marie Curie Initial Training Network Neural Engineering Transformative Technologies (NETT), project number 289146 and through the European Joint Doctorate Complex oscillatory systems: Modeling and Analysis (COSMOS), project number 642563 (TK).}

Published: 18 December 2015

\footnotetext{
* Correspondence: mario.mulansky@isc.cnr.it

Institute for Complex Systems, CNR, Sesto Fiorentino, 50019, Italy
} 


\section{References}

1. Tiesinga P, Fellous J-M, Sejnowski TJ: Regulation of spike timing in visual cortical circuits. Nature Reviews Neuroscience 2008, 9:97-107.

2. Shlens J, Rieke F, Chichilnisky EJ: Synchronized firing in the retina. Current opinion in Neurobiology 2008, 18(4):396-402.

3. Kreuz T, Haas JS, Morelli A, Abarbanel HDI, Politi A: Measuring spike train synchrony. J Neurosci Methods 2007, 165:151.

4. Kreuz T, Chicharro D, Houghton C, Andrzejak RG, Mormann F: Monitoring spike train synchrony. J Neurophysiol 2013, 109:1457.

5. Kreuz T, Mulansky M, Bozanic N: SPIKY: A graphical user interface for monitoring spike train synchrony. ArXiv 2015, 1410.6910v2 (Submitted to JNeurophysiol).

6. Mulansky M, Bozanic N, Sburlea A, Kreuz T: A guide to time-resolved and parameter-free measures of spike train synchrony. Arxiv 2015, 1502.02027. (Submitted to IEEE).

7. Chen Y, Zhang H, Wang H, Chen Y: The role of coincidence-detector neurons in the reliability and precision of subthreshold signal detection in noise. PloS one 2013, 8(2):e56822.

doi:10.1186/1471-2202-16-S1-P133

Cite this article as: Mulansky et al:: Time-resolved and parameter-free measures of spike train synchrony: properties and applications. BMC Neuroscience 2015 16(Suppl 1):P133.

\section{Submit your next manuscript to BioMed Central} and take full advantage of:

- Convenient online submission

- Thorough peer review

- No space constraints or color figure charges

- Immediate publication on acceptance

- Inclusion in PubMed, CAS, Scopus and Google Scholar

- Research which is freely available for redistribution

Submit your manuscript at www.biomedcentral.com/submit 